\title{
Du Campement au camping
}

Une précarité désirée

From Camping to camp-site

\section{Olivier Sirost}

\section{(2) OpenEdition}

Journals

Édition électronique

URL : https://journals.openedition.org/tc/5432

DOI : $10.4000 /$ tc. 5432

ISBN : 1952-420X

ISSN : 1952-420X

Éditeur

Éditions de l'EHESS

Édition imprimée

Date de publication : 5 septembre 2011

Pagination : 98-113

ISBN : 978-2-7351-1410-8

ISSN : 0248-6016

Référence électronique

Olivier Sirost, «Du Campement au camping », Techniques \& Culture [En ligne], 56 | 2011, mis en ligne le 30 novembre 2011, consulté le 29 septembre 2022. URL : http://journals.openedition.org/tc/5432 : DOI : https://doi.org/10.4000/tc.5432 


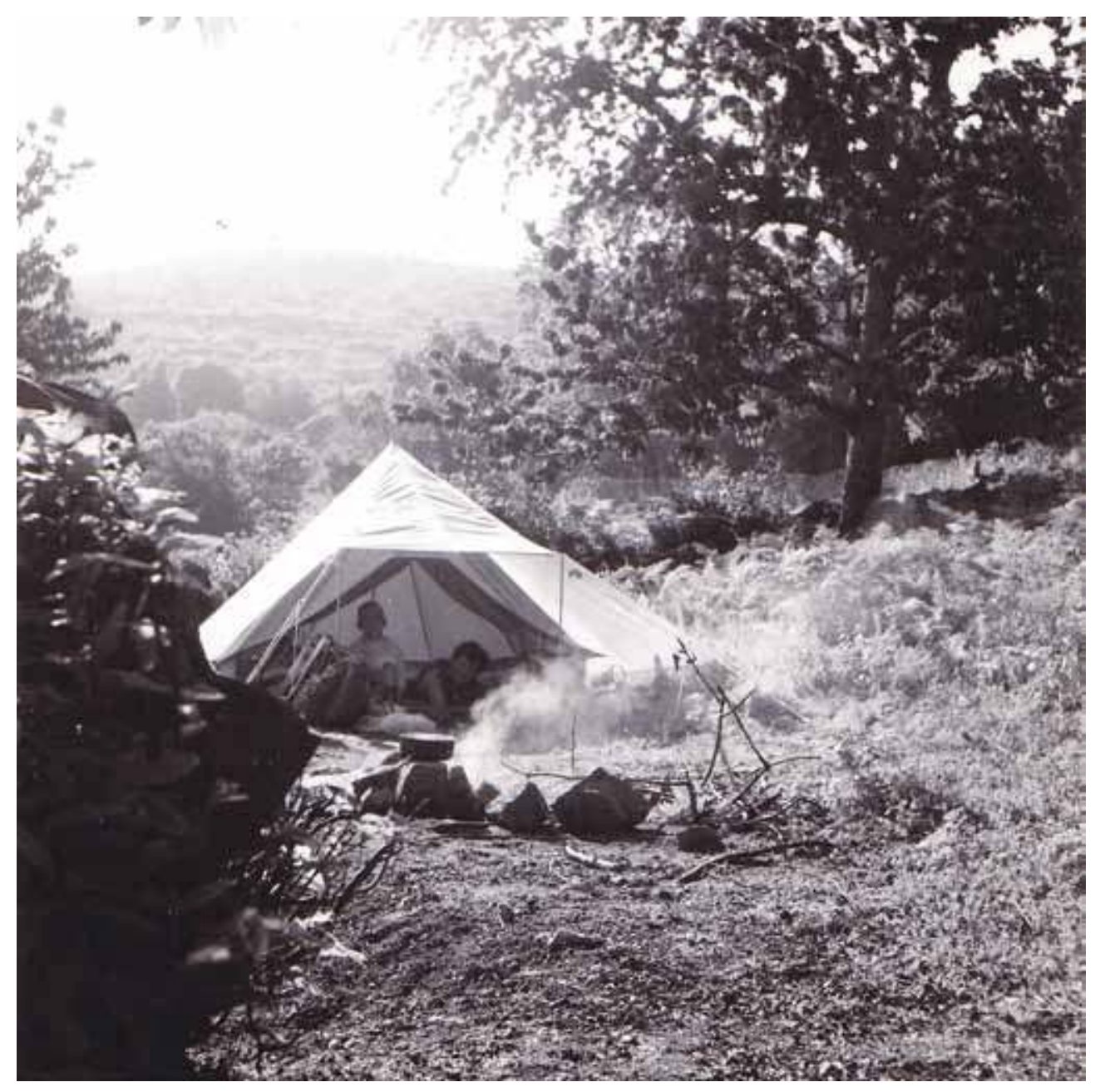

${ }^{\odot}$ Fonds Louis Montagne, coll. 0. Sirost 


\section{DU CAMPEMENT AU CAMPING}

\section{Une précarité désirée}

Le camping est devenu à partir de la fin des années 1960 un dispositif majeur d'encadrement des vacances des Français. La France reste aujourd'hui le pays du camping avec ses 10 millions de pratiquants (dont 3 millions d'étrangers), son parc de près de 9000 terrains et ses innovations techniques émanant d'un réseau de professionnels constitué au début des années 1930. Par sa vaste gamme de formules vacancières le camping existe pour tous les goûts: nomades adeptes du campingcar, de la randonnée et de la caravane, sédentaires résidents dans les divers habitats légers de loisirs (chalets, mobile-homes, cabanons, ... ), adeptes du confort (hébergés dans les parcs résidentiels de loisirs) ou chercheurs de nature s'aventurant dans des trekkings sportifs, collectivistes (Groupement des Campeurs Universitaires, naturistes, camps de jeunes) ou chercheurs de distinction (label qualité, campeurs châtelains). Le camping connaît également ses moments de réversibilité où l'habitat temporaire se transforme en ultime recours précaire d'hébergement et où la tente devient emblème de luttes sociales.

Dans ses origines comme dans son développement le camping est porteur d'une culture technique ${ }^{1}$ autour de l'habitat alternatif et temporaire, au point d'en faire tour à tour un outil de conquête coloniale, un projet pédagogique de sortie en plein air, un projet de société structurant les temps libres, une industrie organisée et majeure des temps libres. 
Les débuts du camping et des excursions bourgeoises. (Fig. 1)
LA GRANDE VOGUE DU CAMPING EN ANGLETERR
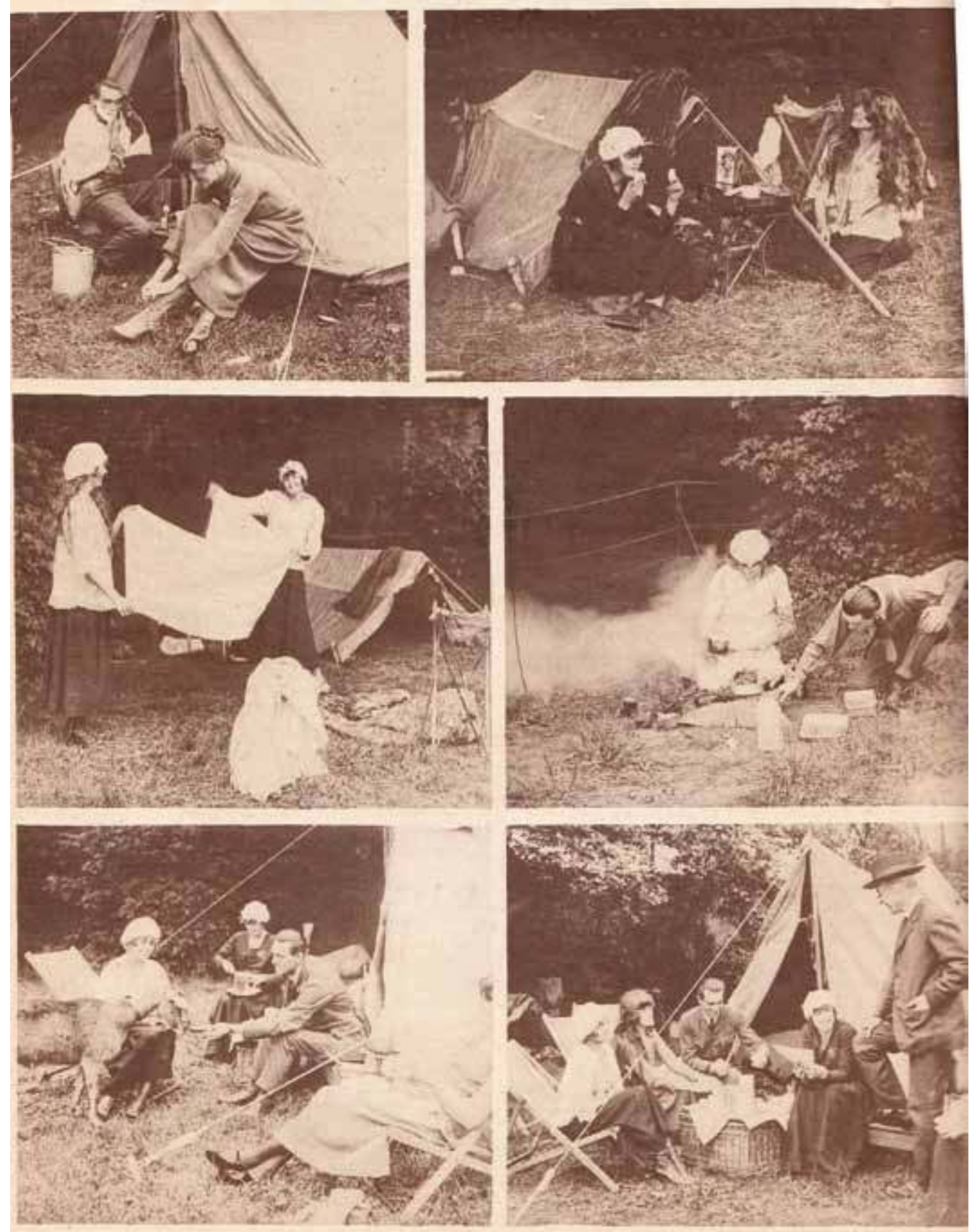

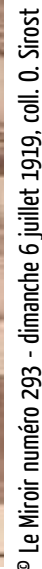




\section{Habitats précaires, habitats temporaires: évolutions techniques, contextes sociaux}

Les origines du camping sont protéiformes. Rappelons que M. Mauss (1968 vol.2: 129) et E. Durkheim (1975 vol 3: 227) voyaient dans le campement une forme d'organisation sociale fondamentale qui traverse les cultures et les sociétés et dont la vieille Europe s'empare à travers l'émergence des mouvements de jeunesse dans un contexte paramilitaire. Cette observation pose d'emblée le caractère labile et réversible d'un abri conçu comme habitat pour nombre de populations nomades transposé dans un contexte loisible de sociétés en voie d'industrialisation (Figure 1).

\section{Les voyages commerciaux et d'exploration XVIII e et XIXe siècles}

Vivre sous la tente, en roulotte ou baraque se voit conférer un nouveau sens dans le cadre des voyages d'explorations coloniaux aux XVIII ${ }^{e}$ et XIX ${ }^{e}$ siècles. Le développement des comptoirs commerciaux à destination de l'Occident (avec notamment les compagnies des Indes) incite les fabricants de mobilier à commercialiser des objets techniques démontables et transportables tout en conservant leur élégance à destination d'une élite sociale dont le métier des affaires et de la politique passe par le voyage. Les malles, les cantines, les secrétaires et bureaux, les cabinets de toilettes, les lits, les tables, les chaises et fauteuils, et mêmes certaines commodes et bibliothèques font l'objet d'une érudition et de trésors d'inventivité pour les rendre transportables et résistants aux affres des déplacements d'affaires. En Angleterre, plusieurs officines se développent en se spécialisant dans le mobilier de voyage. Les manufactures Morgan \& Sanders, William Pocock, Thomas Chippendale, A. Hepplewhite \& Co., et bien d'autres deviennent les inventeurs/fournisseurs officiels de l'empire Britannique et de son expansion coloniale (Brawer 2001).

Si sous le toit on se pose des questions techniques relatives au confort et au style, les fabricants contractualisés avec l'empire Britannique se questionnent également beaucoup à propos du toit, du plancher et des arnachagements ou manières d'acheminer le matériel de la domus en voyage. La toile de tente (ou le canvas) confectionnée dans la même étoffe que les voiles de navigation peut être agrémentée d'un plancher et plus ou moins spacieuse (grâce à un système de laçage) selon les circonstances du périple. Elle se plie, se roule et on la transporte alors à dos d'éléphants ou de chameaux. Les fabricants de meubles concourent pour les marchés proposés par l'armée britannique afin d'équiper les officiers en tentes et accessoires portatifs. Si au début du XviII ${ }^{e}$ siècle les trouvailles restent assez massives et encombrantes se résumant souvent à des meubles démontables qui équipent également les cabines des navires, avec les différents fronts militaires et l'empire victorien c'est l'invention pliable qui est privilégiée. La guerre de Crimée (18531856) marque l'apogée des fournitures portatives pour les tentes d'officiers que l'Empire liste comme pour une naissance (Brawer 2001 : 182). C'est aussi dans ces années que la peinture orientaliste (Thorthon 1998) donne à voir un canvas s'apparentant avec le style de vie du sportsman. Il devient fréquent d'observer devant les campements aux Indes, en Afrique ou aux Amériques, de jeunes anglais affichant une tenue de sport blanche et s'adonnant à quelques parties de cricket ou de football (Thornton 1990). Le tout est 
complété par les parties de chasse et traversées de milieux naturels à caractère panthéiste. L'East India Company va même jusqu'à se charger de la confection de matériel, louant sur place tentes et cabanons à destination des touristes, explorateurs, marchands et hommes d'affaires. Cette organisation va de pair avec l'essor des vacances pour les élites sociales qui choisissent des destinations exotiques (Peltre 1995). La deuxième partie du xixe siècle tranche ainsi avec le déploiement de nombreux porteurs acheminant par « caravanes » le mobilier des occidentaux en voyage.

Les premières pistes de confection du matériel s'offrant au chercheur viennent du caractère polyvalent du mobilier marin. Les voilures, embarcations légères, mobiliers de cabines sont aisément convertis en matériel de campement. Un cahier des charges à destination des fabricants met l'accent sur le caractère d'aménagement d'espaces restreints et d'adaptabilité du matériel à des milieux variés et à des exigences de déplacement (Baubérot \& Bourillon 2009 : 211-225). Il en va de même des troupes de la Grande Armée dont la préparation au bivouac et au cantonnement forge une culture technique de la vie au grand air, notamment expérimentée au camp de Compiègne. Les officiers de Bonaparte ont parfois le luxe de dormir et se réunir sous les tentes marquises, leur mobilier portatif et les nombreux accessoires de voyages acheminés par malles. Les troupes se contentent d'un trou rempli de fourrage et d'une bâche tendue au-dessus de leur tête. Les conquêtes militaires de l'Afrique du Nord amènent les voyageurs occidentaux à découvrir des manières temporaires d'habiter particulièrement efficaces. L'observation de la vie des souks et sous les guitounes se superpose à la capitale itinérante sous toile d'Abd el-Kader dont la smala résista quinze ans au corps expéditionnaire français. L'expérience douloureuse incitera A. De Courtigis à revoir le matériel de campement militaire français (en particulier la tente abri et la tente bivouac) afin de le rendre plus opérationnel en milieu hostile. La tente de l'opposant Abd el-Kader sera exposée au grand public à Paris en guise de trophée pendant plusieurs mois. Il s'agit là d'une véritable entreprise de médiatisation de l'habitat temporaire et mobile ramené par le duc d'Aumale à Chantilly et emblématisé dans les œuvres de Delacroix, Decaen et Vernet ${ }^{2}$. On peut dire au regard des traces liées aux œuvres picturales, que le campement de voyage en Orient, ainsi que le cantonnement militaire, font l'objet d'une attention soutenue dans les années 1870. Au-delà d'un havre de refuge face aux assaillants militaires ou aux éléments naturels déchaînés, le campement devient un espace loisible. On y voit tour à tour des soldats jouer au loto, aux cartes, échanger du tabac ou un café, bivouaquer en montagne ou apprendre à nager dans un cours d'eau, s'offrir une sieste crapuleuse ou chahuter ensemble (Robichon 1998). Le phénomène est sans doute à lier aux projets d'aménagement de l'espace sous le règne de Napoléon III. L'Égypte et plus largement l'Afrique du Nord deviennent des sites touristiques prisés, tout comme la mode de la villégiature. La tente, la cabine, le cabanon, la pergola deviennent des objets qui socialement s'affichent et témoignent de l'appartenance à la classe des loisirs théorisée sous la plume de Thorstein Veblen (1970). La puissance symbolique de l'étoffe et de la tente reste un élément important dans la dynamique du camp militaire et dans la diffusion du style de vie du sportsman. Elle témoigne de l'affichage de sa condition. On en oublie toutefois souvent la " part magique ${ }^{3}$ attachée à l'objet technique quand il a un statut plus pérenne d'habitat.

C'est ce que révèlent aussi les manières de diffuser les résidus ${ }^{4}$ de campement ou de caravane à l'heure de l'avènement des loisirs. Si la tente ou le cabanon s'offrent à voir en tant que prises militaires, ces habitats alternatifs ornementent les grandes villes occidentales à l'occasion des expositions universelles et coloniales, mais également les salons mettant 
en scène les prodigieuses inventions industrielles. Ils apparaissent à travers les différents spectacles (barnums, cabarets, foires...). Avant les succès de la Revue Nègre à Paris et de l'exotisme exhibé par exemple au Paradis Latin ${ }^{5}$, les huttes, tentes et cabanons font partie de la mise en scène des puissances coloniales. Lorsqu'en 1867 Paris accueille l'exposition universelle, les spectateurs peuvent voir de véritables villages indigènes reconstitués et surtout visiter le matériel de campement destiné aux explorations et conquêtes militaires. On y retrouve les établissements élaborant ce type de matériel destiné au parti colonial français (Michel \& $C^{\text {ie }}$ 1872). La tente baraque démontable en 5 ou 8 colis, le manteau individuel du soldat transformable en bâche ou poncho sont les innovations marquantes de l'exposition. On retrouvera ces habitats temporaires utilisés à la belle époque dans les débuts mouvementés des premières associations d'excursionnistes et de campeurs (Sirost 2002 : 49-64). Les excursions élégantes organisées à Luchon ou Fontainebleau pour les lectrices de la revue Fémina font appel à une caravane de plusieurs porteurs pour installer le sweet home entoilé avec un vrai plancher, des lits de camp, un cabinet de toilette portatif, une table et des chaises. La presse de l'époque telle que La vie au grand air, la revue du Touring Club de France, Le Miroir, L'Illustration... présentent tour à tour la mode du campisme, période de transition entre le campement des militaires et le tourisme sous la toile des anglo-saxons.

\section{Renversements de perspectives: confort de vie dans le précaire et durabilité des temps de loisirs}

Cette bourgeoisie coutumière de la villégiature utilise fréquemment les tentes marquises, les pergolas italiennes et abris de chasse dans le cadre des parties de campagnes prolongées. Les fabricants de mobiliers de jardin trouvent dans ces abris en toile un débouché commercial intéressant, quelques officines telles que Monneret ou Monjardet se spécialisent. On retrouve ces fabricants liés à partir de 1904 au Touring Club de France et au récent Canoë club dont ils constituent les membres actifs. Aux côtés des sociétés d'excursionnistes et de préparation militaire qui s'adonnent à la vie au grand air sous la toile, se joignent alpinistes, cyclotouristes, automobilistes et canoéistes. Afin de prolonger la durée de la sortie dominicale, tous souhaitent développer un matériel adapté aux loisirs sportifs. Les inspirations sont multiples. Les sportsmen de l'Alpine Club tels que Mummery ou Whymper élaborent dès la fin du XIX ${ }^{e}$ siècle de nouveaux modèles de tentes légères et aisément transportables. Ces petites merveilles se montrent dans ces camps d'exhibition ou à travers différents salons liés aux nouveaux modes de vie industriels. C'est d'ailleurs lors d'une de ces exhibitions qu'un français ébahi demande à un anglais ce qu'est cet habitat. Le campeur répond au visiteur: « it is a ... tent ». Le français ne retiendra que le début de la phrase et c'est ainsi que naît la tente «Itisa » (Raveneau \& Sirost 2011), première tente légère (Figure 2) adaptée aux

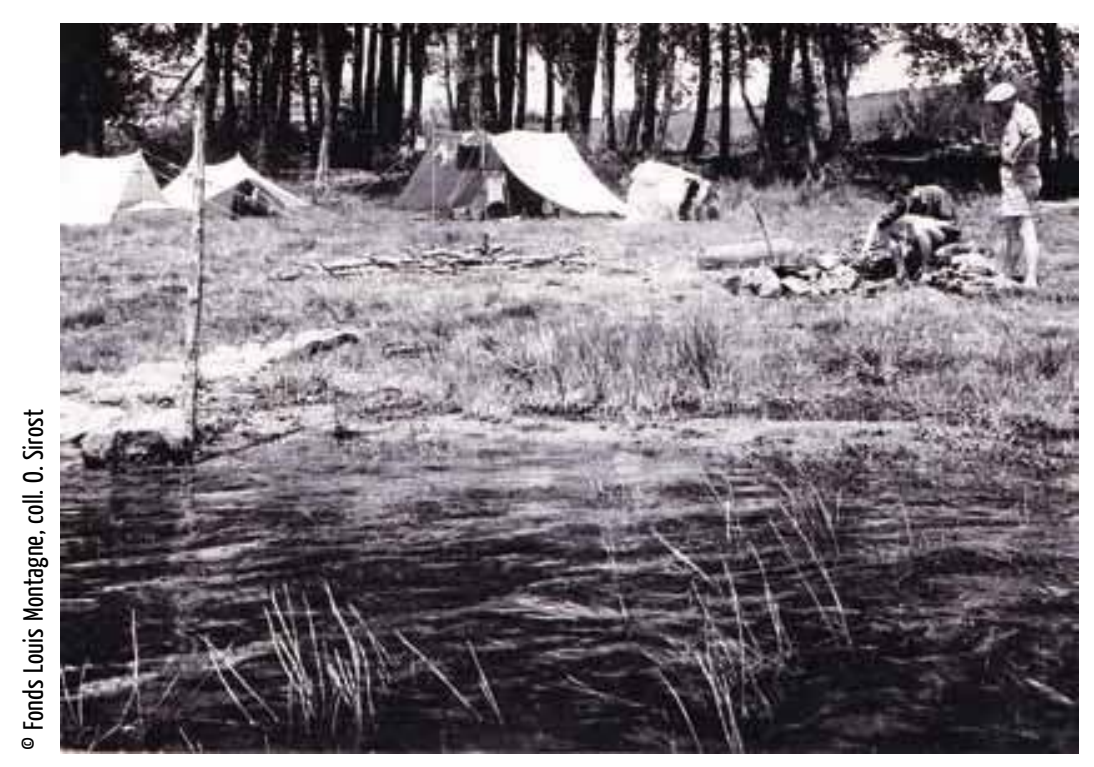

Camping au Mont-Dore (Puy de Dome), 1962, en tente Itisa. (Fig. 2) 


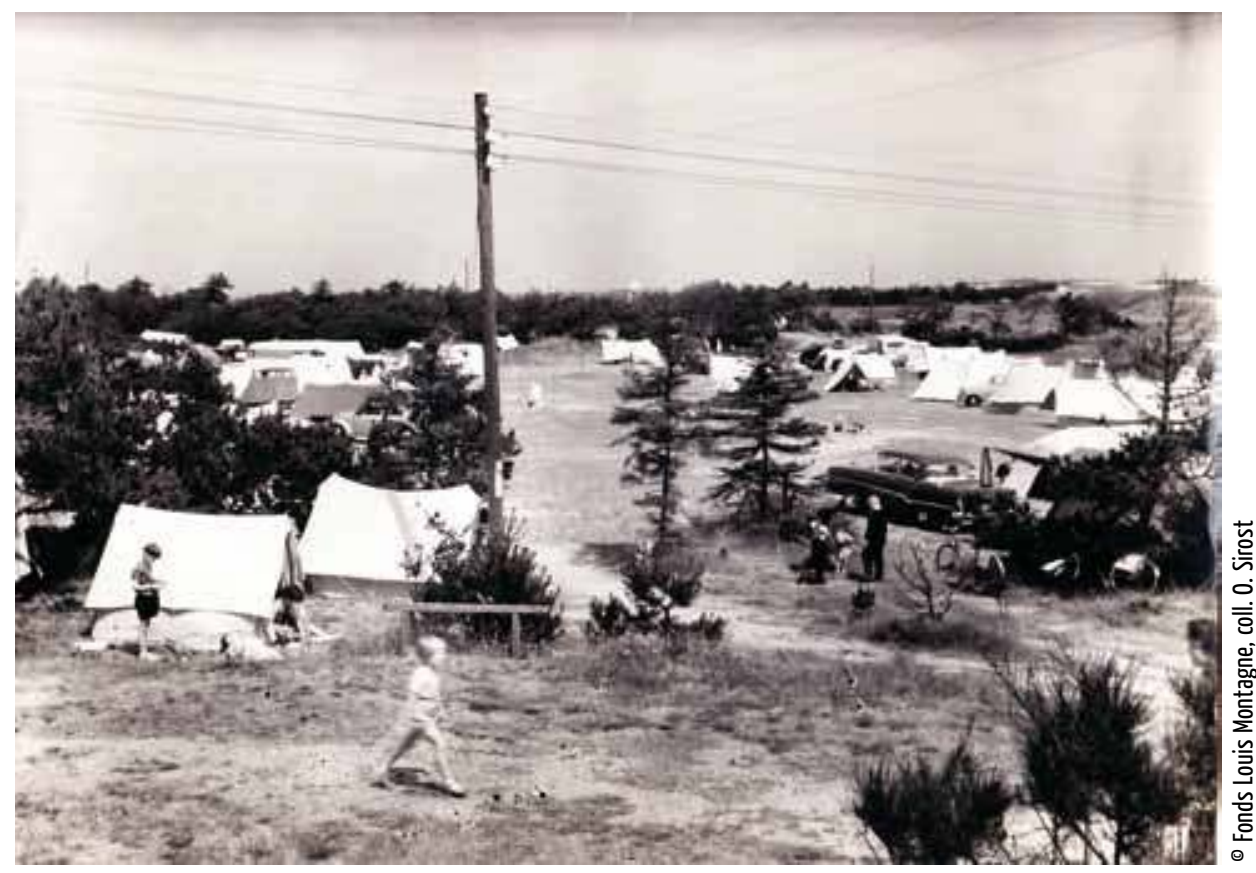

Camping familial

sauvage en Suède dans les années 1950.

(Fig. 3) sports de déplacement. D'autres adeptes de la vie au grand air préfèrent s'adresser directement aux occupants des habitats alternatifs (Sirost 2009a: 211-225). C'est le cas des bourgeois du Touring Club qui visitent directement les roulottes des gens du voyage, étudiant les architectures de ces maisons mobiles et les transformant en habitats de loisirs. Ces « paquebots de la route » ont bien du mal à être tractés par une industrie automobile naissante. Telle comtesse se fait poser un piano dans sa luxueuse roulotte tandis que telle autre offre à ses visiteurs un salon luxueux incluant le service à thé. Catherine Bertho-Lavenir parle de distinction à l'envers pour désigner cette réversibilité de l'habitat temporaire et précaire à des fins loisibles (Bertho-Lavenir 1999 : 304-305). On peut effectivement voir que la tente ou la roulotte des débuts du camping est un amplificateur des économies corporelles. Le passage de la petite tente individuelle, à la tente carrée familiale (modèle qui s'impose dans les années 1950 - Figure 3), puis à la caravane en 1979 (Figure 4 - elle représente alors plus de 50 \% de l'habitat privilégié par les campeurs) et au mobile-home dans les années 1990 montre la sortie progressive d'une logique de campement pour aller vers une hôtellerie de loisirs plus pérenne.

Ce renversement n'est pas le seul phénomène constatable. Les temps de paix et le rapatriement de troupes militaires coloniales éclairent également les débuts des loisirs sous la tente ou en roulotte. Un certain nombre de militaires coloniaux trouvent une reconversion professionnelle dans le métier de garde champêtre. Ce sont les premiers représentants de l'État qui mesurent l'intrusion de touristes dans les corps de fermes, forêts domaniales ou lieux de villégiature des familles de pêcheurs. Ils peuvent également voir les premiers adeptes du camping fréquenter les espaces de fêtes de la nature recensés par les enquêtes des folkloristes et transformer les lieux par l'édification temporaire de villages de toiles. Ces escapades dans la nature voisine et ces robinsonnades font d'ailleurs souvent l'objet de visite des officiers de police, représentants du maire ou du préfet. La rigueur militaire de tenue du camp comme la présentation de carte d'ancien militaire, favorise les relations diplomatiques entre campeurs et institutions dans cette période des débuts du camping. Il n'est pas étonnant de trouver nombre de sociétés de préparation militaire à destination d'un jeune public proposant ce loisir nouveau au début du xx siècle. Il en va de même des premiers mouvements de jeunesse organisés et en particulier du scoutisme. Notons que Robert Baden-Powell, inventeur du mouvement, deviendra président d'honneur 
du Camping Club de Grande Bretagne et d'Irlande. Bref, la confrontation à la nature via l'usage de la tente devient une véritable école de la vie sauvage particulièrement prisée par les pédagogies nouvelles (Daniel \& Pociello 2000). Le sauvage et le sauvageon trouvent ici une transposition particulièrement forte, nous y reviendrons. Toujours dans cette perspective, les récits de retour au pays des écrivains voyageurs dressent une drôle d'analogie entre

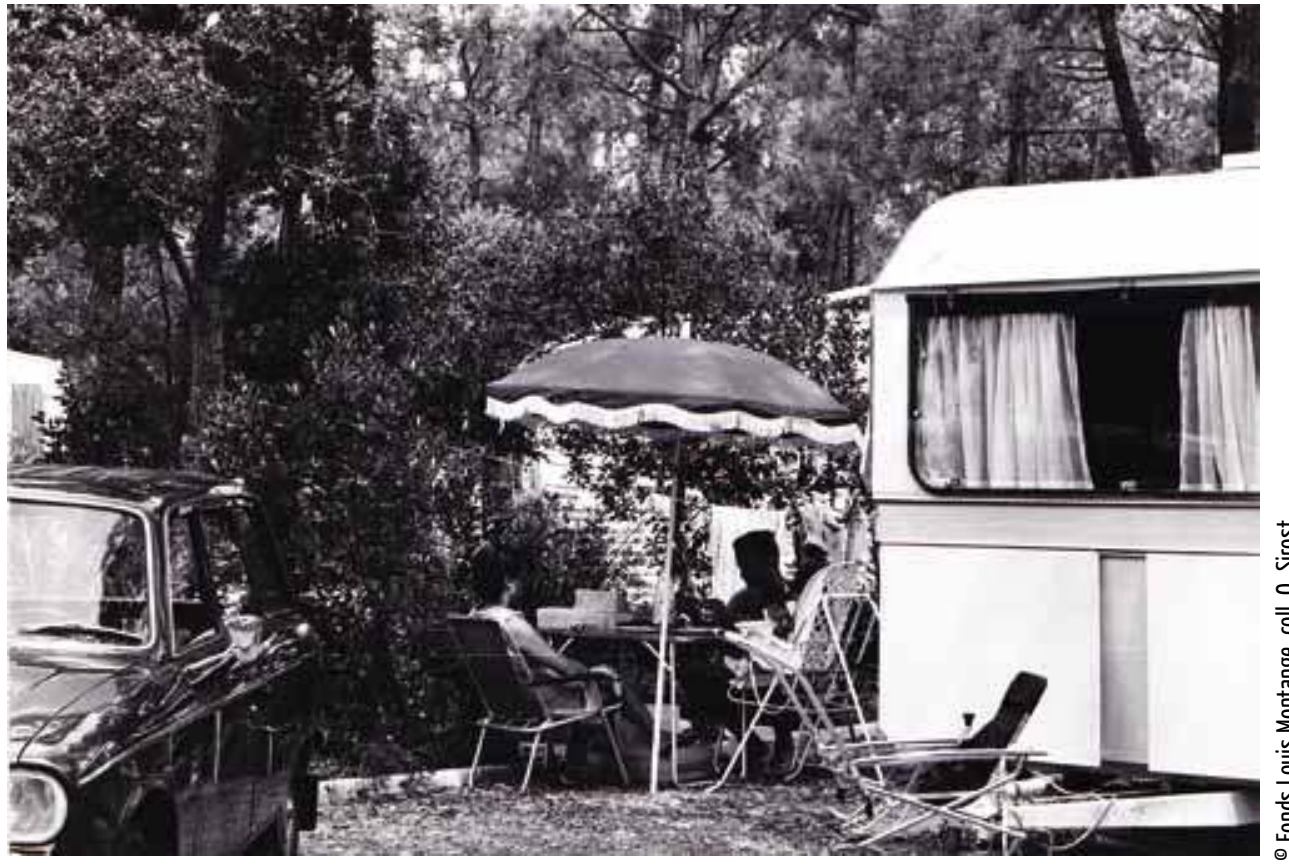
les populations désœuvrées des faubourgs des grandes villes et les

Camping familial en caravane sur terrain organisé, Seignosse Le Penon (Landes), Années 1960.

(Fig. 4)

peuples colonisés. C'est ce que nous révèle ce roman de Dickens, (1937-1938) lorsque de retour à Londres l'écrivain note à travers l'observation des huttes, cabanes et tourbières un air de colonies contracté en Afrique. Lauteur d'Oliver Twist s'inscrit dans une lignée d'intellectuels réformateurs dénonçant les affres de la banlieue naissante.

En Angleterre, le mouvement des cités jardins impulsé par Ebenezer Howard s'accompagne de sorties collectives dans la nature où l'on érige de véritables villages de toile. L'expérience est suivie puis transposée en France par un Leplaysien: Georges Benoît-Lévy. Premier français à adhérer au Camping Club d'Angleterre, Benoît-Lévy est également impulseur du Camping Club Français en 1910 et quelques années plus tard du salon des arts ménagers où l'habitat alternatif connaît un franc succès ${ }^{6}$. L'habitat temporaire est donc au cœur de ses préoccupations. Les premiers articles qu'il publie à partir de 1906 plaident pour la réalisation d'un matériel de campement à la française, s'émancipant alors des importations britanniques (notamment diffusées par le centre franco-américain). C'est à ce moment qu'un vrai débat autour de la culture technique du camping s'installe en France. À la fin du xix siècle, les mouvements de jeunesse parisiens organisent périodiquement des excursions en forêt ou sur le littoral. Comme pour les voyages à pied ou à bicyclette en France à cette période, la nuit se déroule la plupart du temps dans une chambre d'hôtel ou dans la grange d'une ferme. Les plus téméraires s'enroulent dans une couverture à l'abri d'un bosquet ou d'un arbre. Une coopérative d'excursion composée d'une vingtaine de jeunes gens voit le jour en 1903 et commence peu à peu à confectionner son propre matériel. Les expériences sont périlleuses: les tentes baraques construites sur le modèle colonial sont trop lourdes pour les pédestrians; les petites « itisa » ou « bonnet de police » prennent l'eau ou conduisent leurs occupants à l'asphyxie. De plus l'absence de tapis de sol contraint les noctambules à trouver de la paille pour passer une nuit au chaud. 
On emprunte à une mère de famille une machine à coudre pour assembler les pièces, on coupe des branches pour confectionner des piquets ou on se sert des bâtons de marche. Bref l'esprit du temps est à la débrouillardise et au camping spartiate. L'entraide est nécessaire pour profiter pleinement de ce que nombre d'adeptes qualifient de sport. Les manuels de camping mettent alors l'accent sur le concept de camp et de campement, rappelant les bienfaits de l'organisation militaire. En mars 1910, la coopérative donne naissance au Camping club Français (Sirost 2001), antichambre où l'on forme les futurs cadres des éclaireurs unionistes et des éclaireurs de France. On peut donc voir que c'est dans la rencontre entre fabricants et férus de plein air que se développe tout un monde associatif. L'article premier du Camping Club Français consiste à diffuser le goût pour le camping à travers la confection d'un matériel performant et approprié. Ce premier élan reste toutefois cadré par une forte dimension éducative hors l'école. Même les associations laïques telles que le Touring Club de France rappellent leur rôle d'éducation des masses par la nature et le bricolage. Au début des années 1930, la création de syndicats de fabricants et de détaillants de matériel de camping et de plein air consolide une profession. Plus de trois cents magasins spécialisés se développent dans les années 1930 à Paris. Le matériel s'expose dans les salons de l'aéronautique, du nautisme, des arts ménagers. Les revues professionnelles telles qu'Au grand air ou Camping Plein Air augmentent leurs tirages et se diversifient dans l'édition de guides, récits et manuels. Certains fabricants de matériel font même appel à des architectes (comme Roger Tourte par exemple) afin d'élaborer de nouveaux modèles de tentes. Ce développement est suivi également par des entreprises de plus grande importance. La manufacture d'armes de Saint-Étienne voit ainsi dans le matériel de camping une reconversion réussie du matériel militaire. Les grands magasins tels que le Bazar de l'Hôtel de Ville (BHV) ou les Galeries Lafayette développent un rayon spécialisé dans le camping en plein Front populaire. Jacques de Rorthay alors salarié au BHV s'inspire de cette expérience pour fonder en 1940 son premier magasin «Au vieux campeur ». Le camping est donc bien un mouvement urbain et industriel dans ses fondations. Il sert alors pleinement le discours hygiéniste plébiscité par le musée social (legs de la politique sociale de F. Le Play). Les associations de camping portées par le Club Alpin Français et le Touring Club de France organisent alors chaque année, à la demande du musée social, des camps thermaux pour les enfants rachitiques et tuberculeux. Plus de 300000 jeunes profitent chaque été de cette thérapie par le camping dans les années 1930.

Ces différents exemples montrent la grande réversibilité du phénomène. En passant du campement au camping, la vie sous la tente devient une manière de régénérer les populations urbaines de la métropole. Ce sont les habitats alternatifs des colonies qui viennent alors en aide à une société occidentale souffreteuse d'épidémies et d'insalubrité, voyant dans la vie au grand air un salut. Mais le constat ne s'arrête pas là.

Les mots ont ici une importance capitale. Lorsque dans les années 1960 l'État français enquête sur les foules de campeurs se massant sauvagement sur le littoral, les administrateurs utilisent les mêmes termes en forçant leur consonance péjorative. Les campeurs y sont décrits comme « une masse à éduquer, des romanichels, vivant dans un souk, sous la guitoune ou en roulotte » (Sirost 2009b: 204-212). Le film Dupont Lajoie diffusé en 1975, montre comment la vie en camping collectif est une forme de décadence de la civilisation des loisirs, dévoilant des campeurs racistes, aux pulsions sexuelles débordantes et aux encanaillements permanents. La smala c'est eux désormais, dans toute la dimension péjorative du terme. 


\section{Retours à la nature et contextes d'industrialisation: de l'ambiguité des manières d'habiter}

Une autre question est posée par l'habitat temporaire et précaire que suggère le camping. Elle concerne le retour à la nature. À l'instar des communautés hippies des années 1970, le camping se massifie, en particulier à travers ses couches populaires ${ }^{7}$. Face à une société dont certains observateurs disent qu'elle s'individualise, la vie collective offerte par les terrains de camping permet de construire autrement du lien social. Les enquêtes menées par le ministère du tourisme démontrent alors cette recherche de liens amicaux, familiaux ou amoureux facilités par une certaine mise à nu qui dans certaines circonstances frôle la promiscuité. La bande dessinée des années 1970 fait du camping la synthèse des vacances pour tous, forçant sur l'image d'un terreau populaire trouvant là une grande liberté d'expression. La famille O'boulot, les Bidochons, Gaston Lagaffe, en passant par les railleries de Reiser ou San Antonio, incarnent tour à tour une «beaufitude » qui se confond avec la vie sauvage des cités dortoirs. Le cliché n'est pas neuf, il s'actualise à travers l'histoire du camping et nous permet de constater une pensée sauvage bien en dehors de la vie sauvage. C'est ainsi que Joffre Dumazedier (1974 : 78-79) interprète la massification des vacances au tournant des années 1970. Pour aller plus loin, n'y aurait-il pas dans l'habitat spécifique du campeur une mobilisation singulière de cette " pensée sauvage à l'œuvre »? Revenons quelques instants sur les débuts du camping.

Les déserts, les mers et océans, les forêts, les massifs montagneux deviennent des espaces à défi technologique où le bivouac et la tente concentrent les efforts de l'homme face à la nature (Simon 2004). Il apparaît clairement que le matériel de camping s'origine dans ces entreprises. L'étymologie de l'abri vient de la marine et du souci vital pour les voyageurs de trouver un lieu sec, ensoleillé et épargné par le vent. Cette recherche de matrices naturelles où vient se lover l'explorateur fourbu et en proie à la turbulence des éléments naturels trouve un écho particulièrement puissant dans le moment romantique. Les trous, les grottes, les arbres et buissons, offrent des nids spontanés avant que ne se développe le matériel de camping à destination des touristes. Les libertins du xvIII ${ }^{e}$ siècle cultivent par ailleurs ces endroits qu'ils artificialisent dans leurs jeux érotiques (Delon 2000). Les jardins des élites sociales combinent alors ces espaces refuges consacrés aux loisirs amoureux que sont par exemple les labyrinthes végétaux, les grottes artificielles, les abris de jardins (ou dépendances), les chaises à porteurs et les carrosses. Ce modus vivendi n'anticipe-t-il pas la société des loisirs à venir? Il y a dans le camping une manière de renouer avec le chaînon manquant, de rejouer l'homme des cavernes comme le signalait déjà Paul Morand (2001).

L'histoire du camping offre à cet égard des éclairages particulièrement parlants. Un des pionniers des associations de camping et de randonnée, Jean Loiseau, introduit après la Grande Guerre l'indianisme dans différents mouvements de jeunes. Il s'inspire en particulier de l'américain Ernest Thompson Seton et de l'anglais John Hargrave fondateurs respectivement du Woodcraft (science des bois) et du Kibo Kift (faire preuve d'une grande force). Revenant d'un voyage d'exploration naturaliste pour les muséums d'histoire naturelle en 1902, l'ornithologue E.T. Seton découvre sa propriété vandalisée par quelques élèves turbulents du lycée voisin. Il invite alors les fauteurs de troubles à séjourner un week-end en sa demeure pour réparer. Seton enseigne aux trublions les préceptes de la vie des Indiens d'Amérique du Nord qu'il a côtoyé lors de ses voyages et invite ses hôtes 
à dormir sous le tipi. L'expérience sera répétée, ce sont les débuts de ce que plusieurs historiens considèrent comme le scoutisme. Faire de la nature une école de la vie sauvage va être un précepte qui fera recette en occident. Le Woodcraft va connaître de multiples adaptations en Europe. En France c'est Jean Loiseau qui tente d'adapter le concept. Du Club des Jeunes Éclaireurs (en 1911) au mouvement des Compagnons Voyageurs (dans les années 1930), Jean Loiseau cultive cette expérience de vie en petit groupe dans la nature. Ses élèves apprennent le sens de la piste, s'adonnent aux cures de nature néo hippocratiques, et cultivent leur savoir technique du campement (Sirost 2010). Proche des Éclaireurs, Paul Coze travaille au Muséum d'Histoire Naturelle de Paris. Il est spécialement chargé du département des Indiens d'Amérique du Nord. En marge de son activité scientifique il fonde deux mouvements de jeunes: le Cercle Wakanda d'études peau-rouge, et le Club du lasso. Il participe à vulgariser le mythe de la vie sauvage de l'ouest auprès des jeunes français pratiquant le peaurougisme dans les mouvements scouts (Baubérot 1997). Parmi les recrues de Jean Loiseau on note la présence du cinéaste François Truffaut, dont l'expérience vécue lui aurait inspiré son film: L’Enfant sauvage.

Dans cette quête de «primitivisme » ou d'âge d'or, quelques campeurs du Touring Club s'adonnent à une autre passion: l'exploration des grottes et des gouffres. Un groupe de spéléologie émerge alors des campeurs de la grande association de tourisme et entreprend la découverte et le recensement des hauts lieux d'une certaine histoire de l'humanité. Lascaux fait partie des découvertes du groupe qui voyage désormais dans la préhistoire. La revue Grottes et Gouffres est d'ailleurs publiée par les éditions Jean Susse ${ }^{8}$ qui fédèrent l'esprit du camping en France. Les romans préhistoriques écrits par Rosny (auteur notamment de La Guerre $\mathrm{du}$ feu) sont par ailleurs souvent cités dans les revues de camping à partir des années 1940. Ils s'inscrivent dans la propagande du retour à la terre préfigurée par les écrivains champêtres tels que Giono et amplifiée par la politique de Vichy. D’autre part, la pédagogie de la débrouillardise mise en œuvre par le froissartage ${ }^{9}$ comprend parfois l'usage des silex dans l'apprentissage du feu et des techniques de survie dans la nature.

Parmi les éléments de confrontation à la vie sauvage, le camping du début du $\mathrm{xx}^{\mathrm{e}}$ siècle est porteur d'immersions dans la nature et d'une sensibilité écologique. Les premières associations de campeurs, randonneurs et cyclotouristes en Angleterre lancent un mouvement écologiste dont les ramifications vont jusqu'aux Green Shirts et à un socialisme utopique ${ }^{10}$. La fuite loin des villes à des fins de salubrité et par toute forme de transport imaginable permet de renouer avec la médecine hippocratique et l'exposition du corps dénudé aux éléments naturels. Certains comme Jean Loiseau ou Georges Hébert y voient une manière de réveiller le primitif qui sommeille au fond de tout homme civilisé. Pour d'autres, le campeur prend conscience peu à peu du souci de protéger la nature. Comme le dit joliment Jean Hureau dans son manuel de Plein air et camping en 1942, « le plein air commence par un réflexe de midinette qui prend soin de ses plantes chaque matin en ouvrant ses fenêtres ». Laisser son emplacement propre après son bivouac ou lors du démontage du campement devient un leitmotiv d'éducation des campeurs non rattachés à une association dans les années 1950. Ce respect du milieu naturel se double d'un souci de préservation d'un âge d'or du camping que l'on a peur de voir disparaître définitivement. La vie sous tente ou caravane s'accompagne dès lors sur les premiers parcs résidentiels de loisirs (qui voient le jour dans le Var au tournant des années 1960) d'un jardinage et d'un travail d'esthétisation de son emplacement (Figure 5). Les parterres fleuris et les haies entretenues des campeurs annoncent une mise en exotisme des campings haut de gamme des années 1980. 


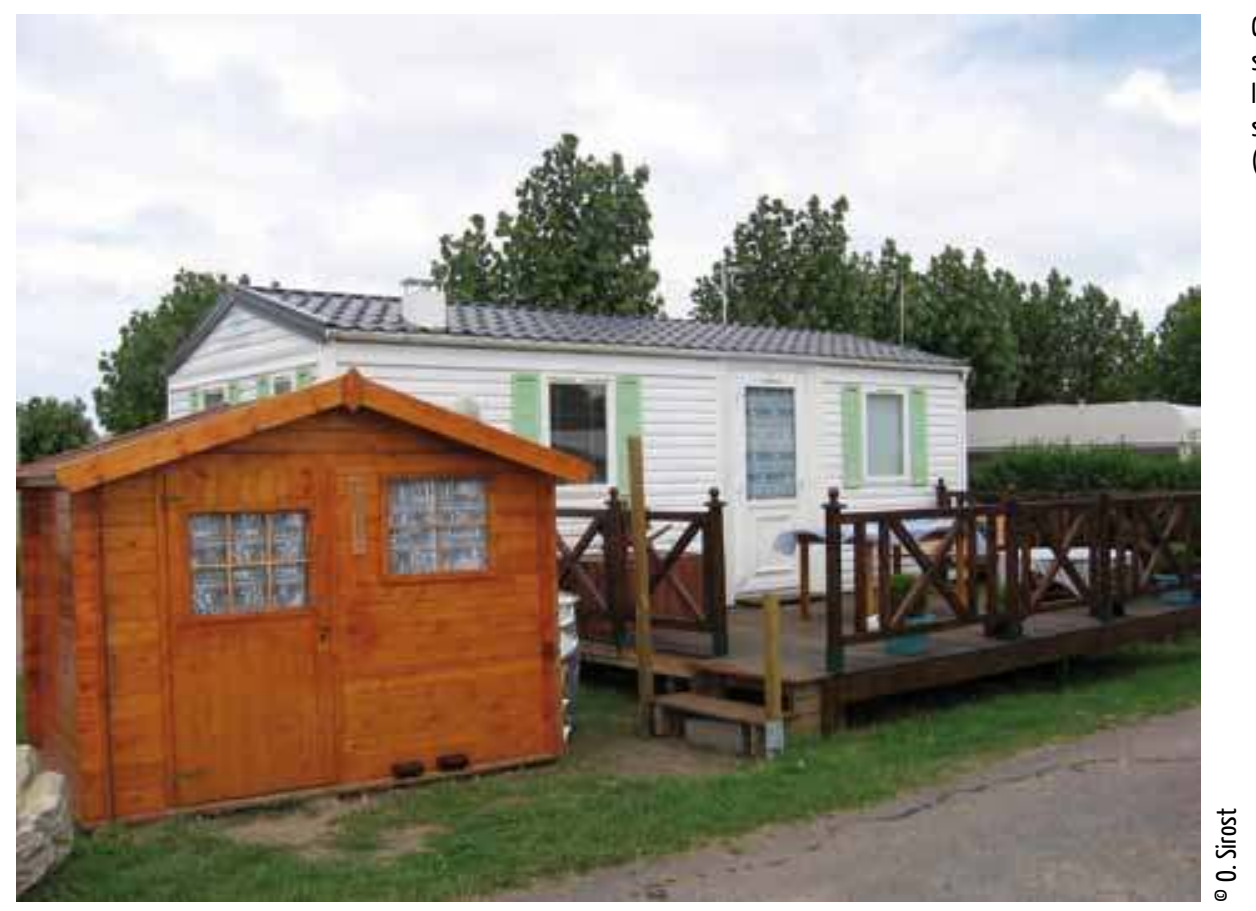

Chalet et abri de jardin symboles du renouveau de I'hôtellerie de plein air, Villerssur-Mer (Calvados), 2006. (Fig. 5)

L'autre dimension sur laquelle vont travailler les promoteurs du camping est psychologique. Dans le contexte de mise en œuvre des pédagogies nouvelles, la vie sous la tente, l'expérience du feu de camp ou de la piste permet de réveiller la bête contenue en soi. Le camping propose tout simplement à l'homme de la ville de se reconnecter avec une pensée pratique, d'anciens schèmes primitifs enfouis sous une disciplinarisation des corps. Le totémisme scout et campeur n'est pas étranger à ce discours. Outre le fait de se mettre dans la peau d'un « primitif » en jouant à l'indien, au néandertalien ou à l'explorateur, le camping est aussi perçu par le politique comme moyen d'encadrement de la jeunesse. En 1962 Maurice Herzog fait des pratiques de plein air un moyen d'insertion des blousons noirs dans les cités dortoirs. Il s'agit d'un apprentissage de la citoyenneté par expérience de la vie spartiate (Barreyre 1992). Enfin, comme pour nombre de revues de plein air, la presse de camping reste un puissant diffuseur d'annonces de rencontres matrimoniales à la fin des années 1930. La promiscuité de la vie sous la tente, l'effort physique et le dévêtissement des corps fait du camping un espace amoureux sans précédent.

Ces réalités multiples, que de manière générique l'on range dans le retour à la nature, sont rendues possible par le camping.

Aujourd'hui la vie sous la tente renvoie à des réalités très différenciées passant par une vraie variété d'habitats temporaires. Le succès commercial des tentes dépliables en 3 secondes, des camping-cars, des chalets en bois ou des habitats traditionnels (yourtes ou tipis) garantissent de beaux jours à une manière temporaire d'habiter la nature. Néanmoins la réversibilité du statut de ces manières d'habiter est omniprésente: tentes de la Croix-Rouge pour «SDF » (Sans Domicile Fixe), expulsions de campeurs Rmistes (personnes touchant le Revenu Minimun d'Insertion) installés à l'année, destruction d'emplacements cabanisés (Figure 6). Enfin, l'implantation des terrains de camping dans 
des espaces de déprises agricoles rend fragiles les hôtelleries de plein air soumises aux inondations et dérèglements climatiques. C'est dans cette tension permanente que se construisent les caractères temporaires et précaires d'un habitat alternatif emblématique des vacances. Comme le disait en son temps A. Van Gennep (1937) le camping est un phénomène sympathique par le fait qu'il ne dure pas. C'est lorsqu'il s'inscrit dans la durée qu'il redevient campement.

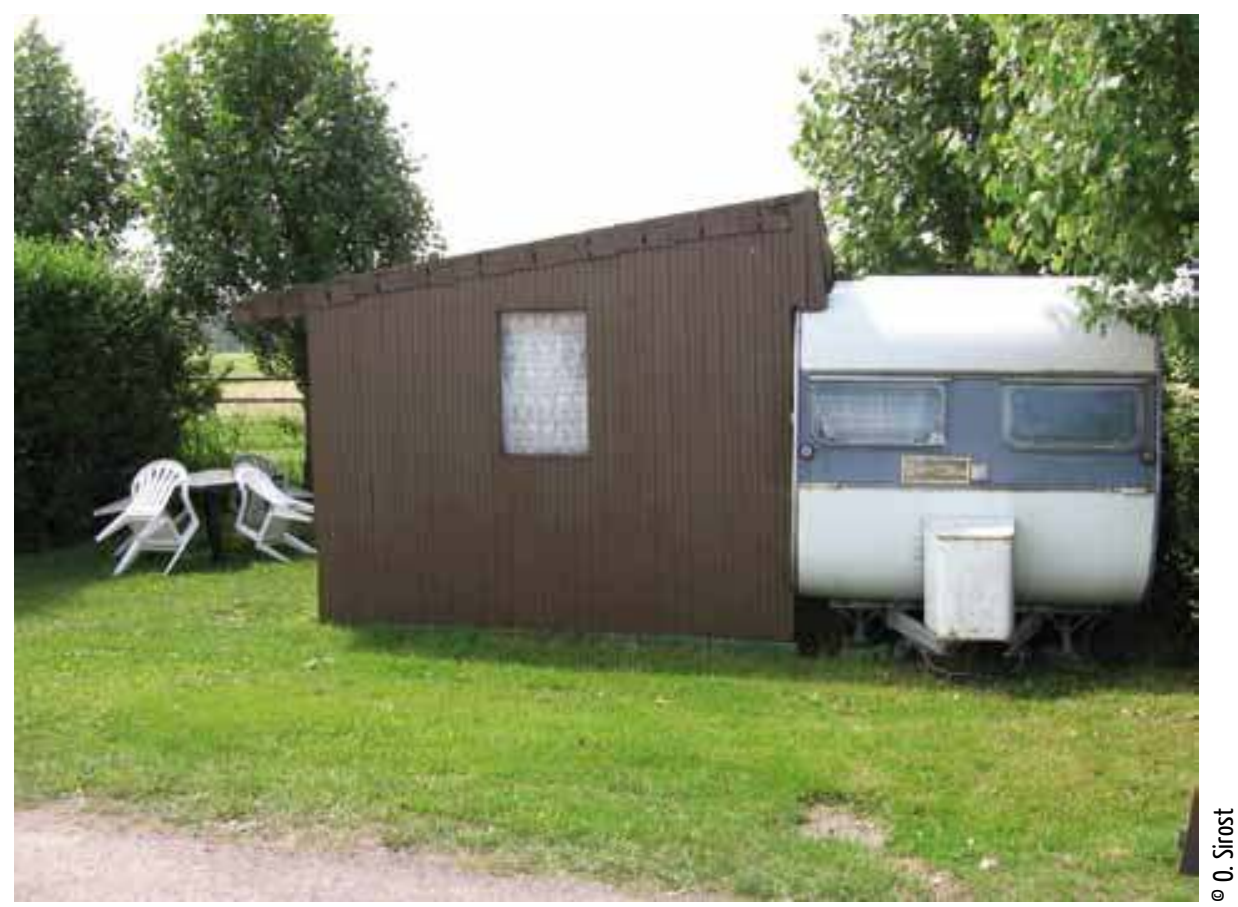

Caravane cabanisée en bord de mer avant procédure d'expulsion, Villers-sur-Mer (Calvados), 2006.

(Fig. 6) 


\section{NOTES}

1. Pour être plus précis, les revues spécialisées de camping qui émergent dans les années 1930 publient tout d'abord les récits de sorties, et les comptes rendus des réunions de clubs où l'on échange les itinéraires et sites de campements, où l'on expérimente et élabore du matériel. Dans les années 1950 ces mêmes revues publient des rubriques spécialisées autour des trucs et astuces, conseils techniques pour l'achat ou la confection du matériel, chroniques de bricolage (Sirost 1999).

2. Coll., Abd el-Kader et l'Algérie au XIX ${ }^{\mathrm{e}}$ siècle, Paris, Somogy, 2003.

3. Au début du XX $X^{\mathrm{e}}$ siècle nombre d'artistes tels que Picasso, André Breton ou Oskar Kokoschka mettent l'accent sur ces objets exposés dans les musées d'ethnographie qui viennent inspirer leurs œuvres. Ils avouent ressentir la manifestation d'une puissance archaique sans être capable de la comprendre. Ce sentiment à l'œuvre sera par la suite explicité notamment par Michel Leiris et Claude Lévi-Strauss.

4. Au sens où l'entend Vilfredo Pareto (Pareto 1968).

5. Le Paradis latin fait partie de ces lieux de divertissement dans la lignée du cabaret et du café-concert où la tente, la hutte et leurs dérivés s'affichent dans la mise en scène de villages coloniaux.

6. Dans Maisons mobiles (Willemin 2004). On y côtoie des maisons en papier ou en carton de l'armée américaine, comme les premières caravanes ou encore les clubs de jeunes de Le Corbusier.

7. Au début des années 1970 un campeur sur deux est ouvrier. Cette distribution disparaîtra dans les années 1980 pour devenir semblable à la distribution des catégories sociales en France. En 1976 la Fédération française de camping et de caravaning lance un plan d'urgence pour tenter de doubler la capacité d'accueil des terrains classés, dépassée par la demande sociale.

8. Jean Susse est un des pionniers du camping en France. Il reprend la tête du Camping club de France dans les années 1930 en rachetant la revue Camping. Il crée les éditions Camping, publiant manuels, topoguides, récits d'aventure et huit autres revues.

9. Technique Scoute de construction et d'installation mise au point par Michel Froissart (Scouts de France) dans les années 1930.

10. Dans la période de l'entre-deux-guerres les mouvements tournés vers le plein air adhèrent pour partie à une vision anti militariste, de partage des ressources et de respect de l'environnement. L'influence protestante explique pour partie l'émergence d'une telle sensibilité qui voit dans les thèses économiques du Major Douglas une alternative au capitalisme montant. On pourra notamment lire Harvey Taylor (1997). 


\section{RÉFÉRENCES}

Barreyre, J.-Y. 1992 Les Loubards. Une approche anthropologique. Paris: L'Harmattan.

Baubéro,t A. 1997, L'Invention d’un scoutisme chrétien. Les Éclaireurs unionistes de 1911 à 1921. Paris: Les Bergers et les Mages.

Baubérot, A. \& Bourillon, F. Urbaphobie. La détestation de la ville aux XIX et XXe siècles. Pompérac: Éditions Bière

Brawer, Nicholas A. 2001 British Campaign Furniture: Elegance under Canvas 1740-1914. New York: Harry N. Abrams.

Bertho-Lavenir, C. 1999 La Roue et le stylo. Comment nous sommes devenus touristes. Paris: Odile Jacob.

Denis, D. \& Pociello, C. 2000 À l'École de l'aventure. Pratiques sportives de plein air et idéologie de la conquête du monde 1890-1940. Voiron: PUS.

Delon, M. 2000 Le Savoir-vivre libertin. Paris: Hachette-Littératures.

Dickens, C. 1837-1839 Oliver Twist, Bentley's Miscellany (Fév. 1837 - avril 1839).

Dumazedier, J. 1974 Sociologie empirique du loisir. Critique et contre-critique de la civilisation du loisir. Paris: Seuil.

Durkheim, E. 1975 Textes. Paris: Minuit.

Mauss, M. 1968 Euvres. Paris: Minuit.

Michel, F. \& Cie 1872 Mémoire concernant le nécessaire de campement. Paris: Impr. Fournier fils.

Morand, P. 2001 Chroniques 1931-1954. Paris: Grasset.

Pareto, V. 1968 Traité de sociologie. Genève: Droz.

Peltre, C. 1995 L'Atelier du voyage. Paris: Gallimard.

Raveneau, G. \& Sirost, O. 2011 Anthropologie des abris de loisirs. Paris: Presses Universitaires de Paris Ouest. Robichon, F. 1998 L’Armée française vue par les peintres 1870-1914. Paris: Herscher/Ministère de la Défense. Simon, M. 2004 Voyageurs. Petite histoire du nécessaire et du superflu. Paris: Hachette.

Sirost, O. 1999 Les Mondes du camping. Marseille: Université de la Méditerranée (Thèse de doctorat).

- 2001 Habiter la nature? Le camping, Revue Ethnologie Française, 2001/4.

- 2002 Habiter en camping ou l'art de se nicher dans le paysage, Communications n 73: 49-64.

- 2009 Du Champêtre au plein air: les débuts du camping en France. In A. Baubérot \& F. Bourillon Urbaphobie. La détestation de la ville aux XIX et XX ${ }^{e}$ siècles. Pompérac: Éditions Bière, 211 - 225.

- 2009 Entre migrations saisonnières et invasions barbares, Cahiers Européens de l'Imaginaire, janvier 2009: 204-212.

- 2009 La Vie au grand air. Aventures du corps et évasions vers la nature. Nancy: Presses Universitaires de Nancy.

— 2010 Les pédagogies de la bonne humeur, Revue Corps, № 8, mars: 41-47.

Thornton, L. 1990 Les Africanistes peintres voyageurs 1860-1960. Paris: ACR Édition.

Thorstein Veblen, T. 1970 Théorie de la classe de loisir. Paris: Gallimard

- 1998 Du Maroc aux Indes. Voyages en Orient. Paris: ACR Édition.

Taylor, H. 1997 A Claim on the countryside. A history of the british outdoor movement. Edinburgh: Keele University Press.

Van Gennep, A. 1937 Le Folklore français. Paris: Laffont.

Willemin, V. 2004 Maisons mobiles. Paris: Alternatives.

Filmographie

Boisset, Y. 1975 Dupont Lajoie. Paris: Sofracima production.

Truffaut, F. 1970 L'Enfant sauvage. Paris: Les Films du Carrosse. 


\section{RÉSUMÉ}

Du Campement au camping. Culture technique héritée des voyages d'exploration et du contexte colonial, l'art du campement s'érige en loisir au cours du xix siècle. Par sa diversité d'habitats-temporaires le camping énonce et renouvelle les formes élémentaires de la vie sociale entre luxe et précarité. C'est la recherche du dénuement, de l'exotisme, du sauvage qui amène le campeur à construire des retours à la nature. Du campisme à l'hôtellerie de plein air cet article dresse une généalogie des manières d'habiter temporairement la nature dans une précarité désirée.

\section{ABSTRACT}

From Camping to camp-site. Technical culture woh provides from explorer's travels in colonial context, art of camp became a leisure in $19^{\text {th }}$ century. With its large scale of shelters, camping contructs elementaries social forms betwin luxury and instability. Quest of nudism, exotism, wildness means nature for campers. Form «campisme» to open air hostel, this issue presents different ways of living temporary in nature.

\section{MOTS CLÉS}

Vie sauvage, camping, campement, exploration, loisirs, jeunesse, abris

\section{KEYWORDS}

Wild life, camp-site, camping, browsing, leisures, youth, shelters 